\title{
Поиск источников ионизации газа в галактиках: дополнение к классическим методам
}

\author{
Д.B. Onapur ${ }^{1}$, A.B. Moucees B.,2,3 $^{1,3}$ \\ 1 Специальная астрофизическая обсерватория РАН, Нижний Архыз, Россия, 369167 \\ doparin2@gmail.com \\ 2 Государственный астрономический институт им. Штернберга Московского государственного \\ университета имени М.В. Ломоносова, Москва, РФ, 119234 \\ 3 Институт космических исследований РАН, Москва, РФ, 117997 \\ moisav@gmail.com
}

Поступила в редакцию 15 ноября 2017 г.

\begin{abstract}
Аннотация. Для изучения состояния ионизованного газа, излучающего в оптическом диапазоне, традиционно применяются диаграммы отношения эмиссионных линий, позволяющие разделить основные источники ионизации: молодые звезды в областях НII, активные галактические ядра, ударные волны. В случае процессов с мощной энергетикой (например, галактик Сейферта) проблем с выделением областей с разным типом ионизации практически не возникает. Однако неопределенность появляется в промежуточных случаях. Открытым остается вопрос о том, чем обусловлено наблюдаемое состояние диффузного ионизованного газа (DIG), в том числе на больших расстояниях от галактической плоскости: утечкой Лайман-квантов из областей звездообразования, старым звездным населением, ударными волнами, порожденными процессом текущего звездообразования. Представляется, что разрешить указанную неопределенность можно добавлением в классические диагностические диаграммы дополнительного параметра - дисперсии скоростей газа по лучу зрения. Мы анализируем наблюдательные данные для нескольких близких галактик с разными источниками ионизации газа: объектов с галактическим ветром, карликовых галактик со звездообразованием. Данные о дисперсии скоростей получены из наблюдений на 6-м телескопе САО РАН со сканирующим интерферометром Фабри - Перо, информация об отношении основных эмиссионных линий взята из опубликованных результатов интегрально-полевой спектроскопии.
\end{abstract}

SEARCH FOR THE GAS IONIZATION SOURCES IN GALAXIES: ADDITION TO CLASSICAL METHODS, by D.V. Oparin, A.A. Moiseev. Emission line ratio diagrams are traditionally used for studying the ionized gas emitting in the optical range. They help to separate main sources of gas ionization like young stars in HII-regions, AGNs, shock waves. Although it makes no problem to mark areas with different types of ionization in case of high energy processes (i.e., Seyfert galaxies), intermediate cases provoke an uncertainty. In particular, it's still unknown what caused the observing state of the diffuse gas (DIG), especially far from the galaxy plane: Lyman-quanta leakage from star formation regions, old star populations or shock waves originated by current star formation. It is supposed possible to overcome this problem by adding an extra parameter to classical diagnostic diagrams - line-of-sight velocity dispersion of the ionized gas. We analyze observational data for several nearby galaxies with different sources of gas ionization: galactic wind or dwarf galaxies with current star formation. Velocity dispersion data are 
obtained with a scanning Fabry-Perot Interferometer (FPI) at the 6-m telescope BTA, while the emission line ratios are taken from the published 3D-spectroscopy results.

Ключевые слова: внегалактическая астрономия, межзвездная среда, карликовые галактики

\section{1 Введение}

Диаграммы отношения оптических эмиссионных линий (ВРТ-диаграммы, Балдвин и др., 1981) - широко распространенный способ диагностики источников ионизации газа. С их помощью можно уверенно разделять области, где наибольший вклад в ионизацию газа вносят молодые ОВ-звезды от, например, областей, ионизованных нетепловым континуумом активного галактического ядра (AGN). В то же время на диаграммах смешиваются области, ионизованные ударными волнами, излучением звезд асимптотической ветви гигантов или ядер галактик типа LINER.

Добавление к классическим диагностическим диаграммам еще одного параметра - дисперсии скоростей ионизованного газа по лучу зрения $(\sigma)$ - дает возможность разрешить эту неопределенность в тех случаях, когда рост $\sigma$ указывает на увеличение турбулентных скоростей газа за фронтом ударной волны. Но для уверенных оценок $\sigma$ необходимо заметно лучшее спектральное разрешение, чем требуется для измерения потоков в отдельных спектральных линиях. Поэтому до недавнего времени зависимость отношения линий, характеризующих ударную ионизацию от $\sigma$, рассматривалась лишь изредка и, в основном, для галактик с достаточно высоким $(\sigma>100 \mathrm{kм} / \mathrm{c})$, таких как галактики с мощным звездообразованием (Монреаль-Иберо и др., 2006; Хо и др., 2014). Подобный подход ранее не применялся для изучения ионизации диффузного газа в карликовых галактиках, вокруг отдельных областей звездообразования в них или же на удалении от галактической плоскости. В то же время идет продолжительная дискуссия об источниках ионизации этого диффузного газа (DIG) в галактиках, на роль которых отводят старое звездное население, утечку Лайман-квантов из областей HII, а также, возможно, ударные фронты, вызванные процессами звездообразования (Джонс и др., 2017).

Для изучения протяженных структур низкой яркости в галактиках наиболее эффективно применять методы панорамной (3D) спектроскопии. Но большинство имеющихся наблюдательных данных по спектрофотометрии и кинематике газа близких галактик (например, обзор CALIFA (Санчез и др., 2012)) получено со спектральным разрешением FWHM $>5 \AA$, что в терминах дисперсии лучевых скоростей составляет более 100 км/с. Это является вынужденным компромиссом при изучении объектов низкой поверхностной яркости.

Наблюдения со сканирующим интерферометром Фабри-Перо (ИФП) позволяют получить в несколько раз лучшее разрешение (правда, в узком спектральном диапазоне), благодаря чему становится возможным построение карт дисперсий скоростей ионизованного газа по уширению профиля эмиссионных линий даже в маломассивных галактиках.

\section{2 Наблюдательные данные}

Наши наблюдения проводились в первичном фокусе 6-м телескопа БТА САО РАН со сканирующим интерферометром Фабри-Перо, установленным в фокальных редукторах SCORPIO (Афанасьев, Моисеев, 2005) и SCORPIO-2 (Афанасьев, Моисеев, 2011). Эмиссионные линии (Н $\alpha$ или [NII] $\lambda 6583)$ выделялись с помощью узкополосного фильтра шириной $\sim 20 \AA$. Наблюдения с различными ИФП обеспечивали спектральное разрешение FWHM =0.4-1.7 А. Масштаб изображений составил $\sim 0.7 \mathrm{arcsec} / \mathrm{px}$ при поле $6.1^{\prime} \times 6.1^{\prime}$. В результате сканирования получался куб данных, где каждый пиксель содержал спектр из нескольких десятков каналов. Наблюдаемые профили эмиссионных линий аппроксимировались функцией Фойгта. По результатам аппроксимации строились карты лучевых скоростей ионизованного газа и карты дисперсии лучевых скоростей. 
Для UGC10043 и Arp212 использовались данные обзора CALIFA, проводившегося с помощью спектрографа PPAK/PMAS (Ротт и др., 2005) на 3.5-м телескопе обсерватории Калар-Альто. Массив световолокон РPAK состоит из 331 элемента диаметром 2.7", собранных в шестиугольное поле $74^{\prime \prime} \times 64^{\prime \prime}$. Использовались данные со спектральным разрешением FWHM $\sim 5-9 \AA$ в диапазоне длин волн 3750-7500 А. Галактика VIIZw403 наблюдалась с мультизрачковым спектрографом MPFS (Афанасьев и др., 2001) на БТА. Массив квадратных линз в сочетании со световолокнами обеспечивал поле зрения $16 \times 16$ элементов с масштабом 1" на линзу. Куб данных, представленный в статье (Архипова и др, 2007), является мозаикой размером $49^{\prime \prime} \times 31^{\prime \prime}$ из семи полей MPFS. Спектральный диапазон составлял 4250-7200 А, разрешение - $8 \AA$.

\section{3 Диагностические диаграммы}

\subsection{UGC10043: галактический ветер}

UGC10043 - видимая с ребра спиральная галактика, удаленная от нас на расстояние $\sim 35$ Мрс. Наблюдения в линиях $\mathrm{H}_{\alpha}$ и [NII], проведенные на HST (Мэтьюс, де Гриджс, 2004), обнаружили признаки звездообразования в галактическом ядре, а также протяженную эмиссионную структуру, перпендикулярную диску, являющуюся результатом воздействия галактического ветра. В работе (Лопез-Коба и др., 2017) нами представлены диагностические диаграммы для центральной части галактики по данным обзора CALIFA. Часть точек на графике, относящихся к центральной области звездообразования, лежит в области, характерной для фотоионизации молодыми звездами, в то время как другие попадали в область, свойственную для ударного возбуждения. В рамках модели ударного возбуждения было наложено ограничение на скорость ветра - не более 400 км/с. Наши наблюдения со сканирующим ИФП в эмиссионной линии [NII] показали, что кривая вращения, амплитуда которой достигает $150 \mathrm{~km} / \mathrm{c}$, вносит основной вклад в лучевые скорости ветровой структуры. Для ее учета была использована простая геометрическая модель, в которой ветровая структура представлена как два вращающихся усеченных конуса (Опарин, Моисеев, 2015). Полученная в рамках этой модели скорость галактического ветра, доходящая до 250 км/с, согласуется с моделью ударного возбуждения газа, построенной на основе данных CALIFA.

На ВРТ-диаграммах представленных на рис. 1 (верхний ряд) видно, что области с ударным возбуждением эмиссионных линий в ветровой туманности характеризуются более высокой дисперсией скоростей в сравнении с областями, где доминирует фотоионизация. При этом наблюдается положительная корреляция между отношениями линий [SII] к $\mathrm{H}_{\alpha}$, [NII] к $\mathrm{H}_{\alpha}$, [OI] к $\mathrm{H}_{\alpha}$ и $\sigma$ (см. pис. 1).

\subsection{VIIZw403: вспышка звездообразования в карликовой галактике}

VIIZw 403 - одна из ближайших ( $\mathrm{D}=4.5$ Мпк) голубых компактных карликовых галактик с несколькими эпизодами звездообразования. Текущая вспышка локализована в центральном килопарсеке, окруженном диском со старым звездным населением. Здесь выделяются несколько компактных ассоциаций ОВ-звезд и связанные с ними расширяющиеся оболочки HII, погруженные в диффузный ионизованный газ (Егоров, Лозинская, 2011).

Дисперсия лучевых скоростей ионизованного газа лежит в диапазоне $\sim 20-40$ км/с. На ВРТдиаграммах большинство точек располагается в области фотоионизации. Некоторое количество точек с более высокой дисперсией оказывается вблизи разделительной кривой.

При этом расширяющиеся оболочки НII, связанные с яркими областями зведообразования, характеризуются меньшим уровнем дисперсии ( 20 км/с) (Моисеев, Лозинская, 2012$)$. Одна из областей с более высокой $\sigma$ располагается между двумя такими оболочками. Другие расположены на периферии диска ионизованного газа (Моисеев, Лозинская, 2012). Мы можем сделать вывод о том, что 
вклад ударного возбуждения в ионизацию газа в данной галактике в целом незначителен и даже на границах расширяющихся оболочек уступает фотоионизации.

\subsection{Arp212: галактика с полярным кольцом}

Arp 212 - пекулярная галактика в которой Моисеев (2008) выделил две кинематически различные вращающиеся газовые подсистемы: внутренний диск размером 3.5 кпк и внешние области HII, орбиты которых располагаются под значительным углом к звездному диску. Наблюдаемая картина объяснялась в предположении, что НІ во внешних областях галактики расположен в широком кольце (диаметром около 20 кпк), вращающемся в плоскости, примерно ортогональной к диску.
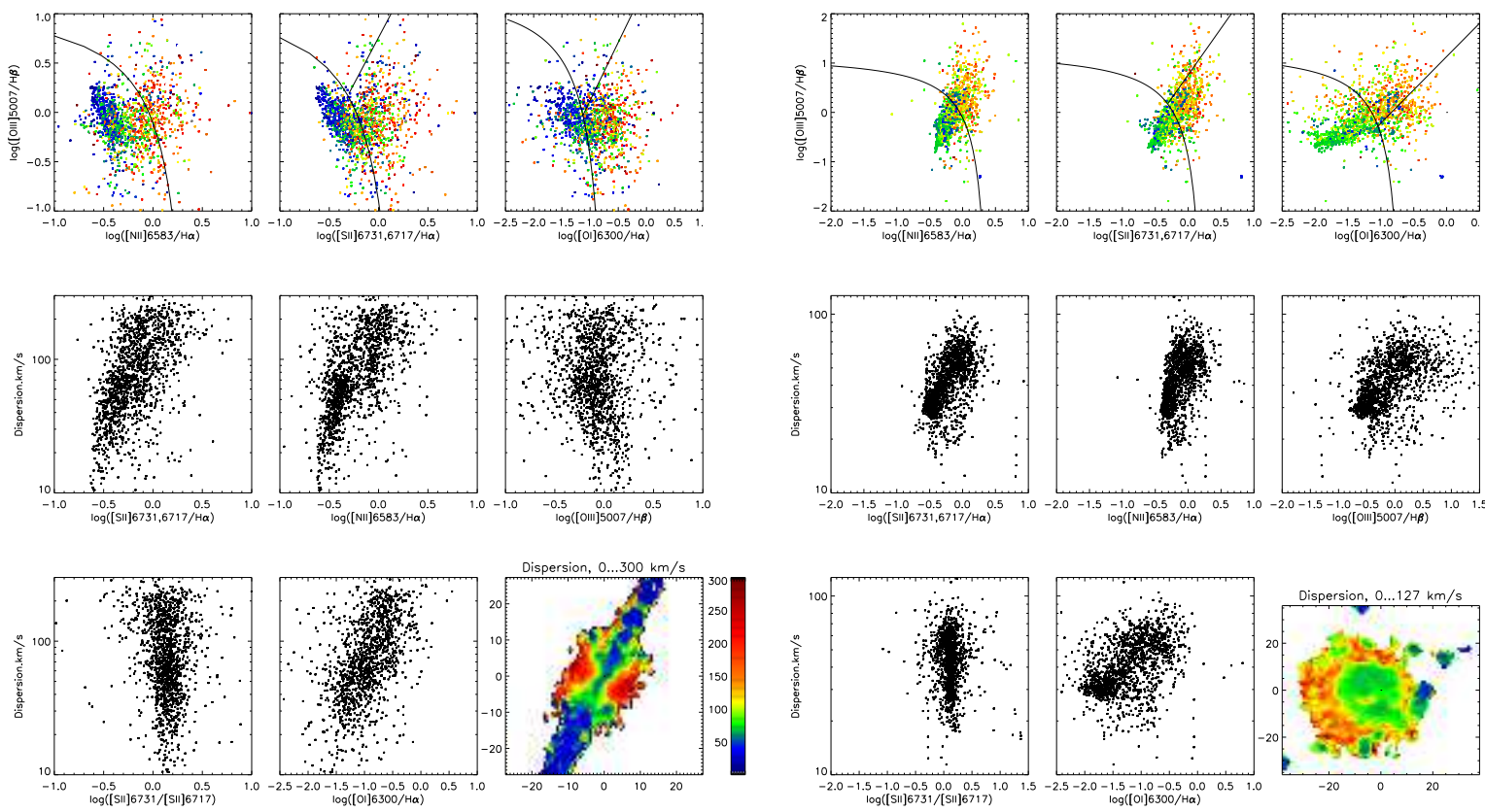

Рис. 1. Левая серия рисунков - UGC10043, правая - Arp 212. Вверху - классические ВРТ-диаграммы. Цветом показана дисперсия скоростей ионизованного газа по лучу зрения в данном пикселе. Линии, разделяющие области с разным типом ионизации, взяты из (Кьюли и др., 2006). Внизу - зависимость дисперсии скоростей от отношения эмиссионных линий. Внизу справа - карта дисперсии лучевых скоростей

По мере уменьшения радиусов орбит газовых облаков угол их наклона уменьшается, и на радиусе 2-3 кпк газ из кольца начинает выпадать в плоскость галактики, провоцируя вспышку звездообразования. Именно здесь наблюдается наиболее высокая дисперсия лучевых скоростей, доходящая до 80-100 км/с (см. рис. 1, справа). При этом точки, относящиеся к этой зоне столкновения газовых подсистем, на ВРТ-диаграммах (рис. 1, справа) смещены от областей с доминированием фотоионизации в сторону области доминирования ударной ионизации. В то же время в центральной части галактики фотоионизация явно доминирует.

Мы попробовали количественно охарактеризовать это смещение, построив графики зависимости $\sigma$ от расстояния до модельной кривой (Кьюли и др., 2001), отделяющей НІІ-области от регионов с другим типом ионизации (рис. 2). Можно видеть, что рост дисперсии скоростей по лучу зрения коррелирует с удалением на ВРТ-диаграмме от зоны, характерной для ионизации молодыми звездами. 


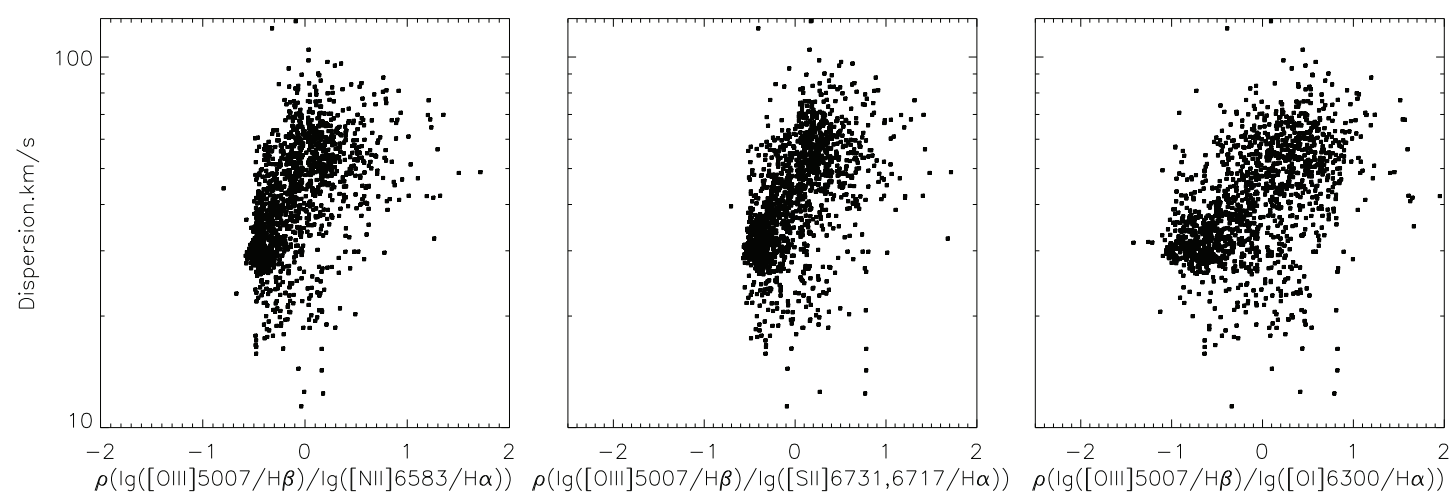

Рис. 2. Зависимость $\sigma$ от расстояния точки до демаркационной кривой на ВРТ-диаграмме, разделяющей области с различными механизмами ионизации (Кьюли и др., 2001). Отрицательная часть смещена в сторону, характеризующуюся фотоионизацией молодыми звездами, положительная - в сторону иных механизмов

\section{4 Заключение}

Наша идея состоит в совместном использовании карт дисперсии скоростей ионизованного газа, полученных из наблюдений со сканирующим ИФП и панорамных данных по спектрофотометрии галактик с низким спектральным разрешением. Наблюдаемая величина дисперсии лучевых скоростей, характеризующей турбулентные движения ионизованого газа, может быть обусловлена разными причинами, такими как вириальные движения в гравитационном потенциале галактики, воздействие на газ расширяющихся оболочек или, в общем случае, - энергией, “впрыскиваемой” в межзвездную среду процессами звездообразования (Моисеев и др., 2015; Крамхольц, Бурхарт, 2016). Также различные причины влияют на величину отношения линий с разным механизмом возбуждения. Объединение этой информации позволяет в ряде случаев сделать однозначные выводы о вкладе ударных волн в ионизацию газа в областях с низкой поверхностной яркостью. Так, только по отношению линий в конической туманности в UGC10043 нельзя однозначно сказать, с чем связан рост относительной интенсивности запрещенных линий - с ионизацией ударными волнами от центральной вспышки звездообразования или со старым звездным населением толстого диска, в котором она расположена. Но добавление информации о кинематике газа говорит о том, что это - современный галактический ветер. В Arp 212 наш подход позволил подтвердить ранее высказанное в работе (Моисеев, 2014) предположение о том, что здесь наблюдается прямое столкновение газовых облаков на наклонных орбитах с основным диском галактики, порождающее ударные фронты.

Работа поддержана грантом Российского научного фонда 17-12-01335.

\section{Литература}

Архипова В.П., Лозинская Т.А., Моисеев А.В., Егоров О.В. // Astron. Rep. 2007. V. 51. P. 871.

Афанасьев В.Л., Додонов С.Н., Моисеев А.В. // Stellar dynamics: from classic to modern / Eds

Osipkov L.P., Nikiforov I.I. Saint-Petersburg: Univ. Press. 2001. P. 103.

Афанасьев В.Л., Моисеев А.В. // Письма в Астрон. журн. 2005. № 319. С. 214.

Афанасьев В.Л., Моисеев А.В. // Baltic Astronomy. 2011. V. 20. Р. 363.

Балдвин и др. (Baldwin J.A., Phillips M.M., Terlevich R.) // Publ. Astron. Soc. Pacific. 1981. V. 93. P. 5. 
Джонс и др. (Jones A., Kauffmann G., D’Souza R., Bizyaev D., Law D., et al.) // Astron. Astrophys. 2017. V. 599. P. 141.

Егоров О.В., Лозинская Т.А. // Astrophys. Bull. 2011. V. 66. P. 315.

Крамхольц, Бурхарт (Krumholz M.R., Burkhart B.) // Mon. Not. Roy. Astron. Soc. 2016. V. 4586. P. 1671.

Кьюли и др. (Kewley L.J., Dopita M.A., Sutherland R.S., Heicler C.A., Trevena J.) // Astrophys. J. 2001. V. 556. P. 121.

Кьюли и др. (Kewley L.J., Groves B., Kauffmann G., Heckmann T.) // Mon. Not. Roy. Astron. Soc. 2006. V. 372. P. 961.

Лопез-Коба и др. (Lopez-Coba C., Sancez S.F., Bitaskis T., Cruz-Gonzalez I., Morisset C., et al.) // Mon. Not. Roy. Astron. Soc. 2017. V. 467. P. 4951.

Моисеев А.В. // Astrophys. Bull. 2008. V. 63. Р. 201.

Моисеев А.В. // Astrophys. Bull. 2014. V. 69. P. 1.

Моисеев А.В., Лозинская Т.A. // Mon. Not. Roy. Astron. Soc. 2012. V. 423. P. 1831.

Моисеев А.В., Тихонов А.В., Клыпин А. // Mon. Not. Roy. Astron. Soc. 2015. V. 449. P. 3568.

Монреаль-Иберо и др. (Monreal-Ibero A., Arribas S., Colina L.) // Astrophys. J. 2006. V. 637. P. 138.

Мэтьюс, де Гриджс (Mattews L.D., de Grijes R.) // Astrophys. J. 2004. V. 128. P. 137.

Опарин Д.В., Моисеев А.В. // Astrophys. Bull. 2015. V. 70. Р. 392.

Ротт и др. (Roth M.M., Kelz A., Fechner T., Hahn T., Bauer S-M., et al.) // Publ. Astron. Soc. Pacific. 2005. V. 117. P. 620.

Санчез и др. (Sanchez S.F., Kennicutt R.C., Gil de Paz A., van de Ven G., Vilchez J.M., et al.) // Astron. Astrophys. 2012. V. 538. P. 31.

Хо и др. (Ho I-T., Kewley L.J., Dopita M.A., Medling A.M., Allen J.T., et al.) // Mon. Not. Roy. Astron. Soc. 2014. V. 444. P. 3894. 